\title{
The Role of the Autonomic Nervous System in Liver Regeneration and Apoptosis - Recent Developments
}

\author{
Takayoshi Kiba \\ Third Department of Internal Medicine, Yokohama City University, School of Medicine, Yokohama, J apan
}

\section{Key Words}

Liver regeneration - Apoptosis - Autonomic nervous system

\begin{abstract}
Hepatocytes have a great replicative capacity and are capable of repopulating the liver. Previous studies have suggested that the autonomic nervous system regulates liver regeneration and apoptosis. Moreover, the central nervous system modulates them through the autonomic nervous system. The lateral hypothalamus (LH) area and the ventromedial hypothalamus (VMH) nucleus have been studied for their role in the integration of neurohumoral information. The $\mathrm{LH}$ is part of the parasympathetic system, while the VMH belongs to the sympathetic system. Lesions of the LH reportedly induced an increase in sympathetic nerve activity, while those in the VMH produced facilitation of vagus nerve activity. Moreover, $\mathrm{VMH}$ or $\mathrm{LH}$ lesions facilitate hepatic regeneration after partial hepatectomy. Furthermore, the hypothalamus mediates hepatic apoptosis through the autonomic nervous system. Although further studies are needed to define the role clearly, the autonomic nervous system is one of the important factors that regulate liver regeneration and apoptosis.
\end{abstract}

Copyright $@ 2002$ S. Karger AG, Basel

\section{Introduction}

The autonomic nervous system influences many of the functions of the body, including those of cardiovascular system, kidneys, liver, pancreas, gastrointestinal tract and glands [1] (fig. 1). Detailed studies of the mechanisms that regulate liver growth and apoptosis have been done in animals subjected to partial hepatectomy or chemical injury [2]. Since the autonomic nervous system directly innervates the hepatic parenchyma and has a role in metabolic control [3], it seems likely that liver regeneration and apoptosis are cooperatively regulated by both humoral factors and the autonomic nervous system. Moreover, it is well known that autonomic abnormalities and neuropathy tend to increase with age [4]. The prevalence and severity of autonomic dysfunction appears to be related to the severity of liver disease and is associated with an increase in mortality [5,6]. It is also reported that patients with liver cirrhosis have parasympathetic hypofunction and sympathetic hyperfunction [7, 8]. In recent years, there has been increasing interest in the relationships between liver and autonomic nervous system. Here, the author reviews the current status of studies aimed at understanding these relationships.
Takayoshi Kiba, MD, PhD

Third Department of Internal Medicine

Yokohama City University, School of Medicine

3-9 Fukuura, Kanazawa-ku, Yokohama 236-0004 (Japan)

Tel. +81 45 5741011, Fax +81 455741075 , E-Mail takkiba@hotmail.com 
Fig. 1. Schematic representation of the auto-

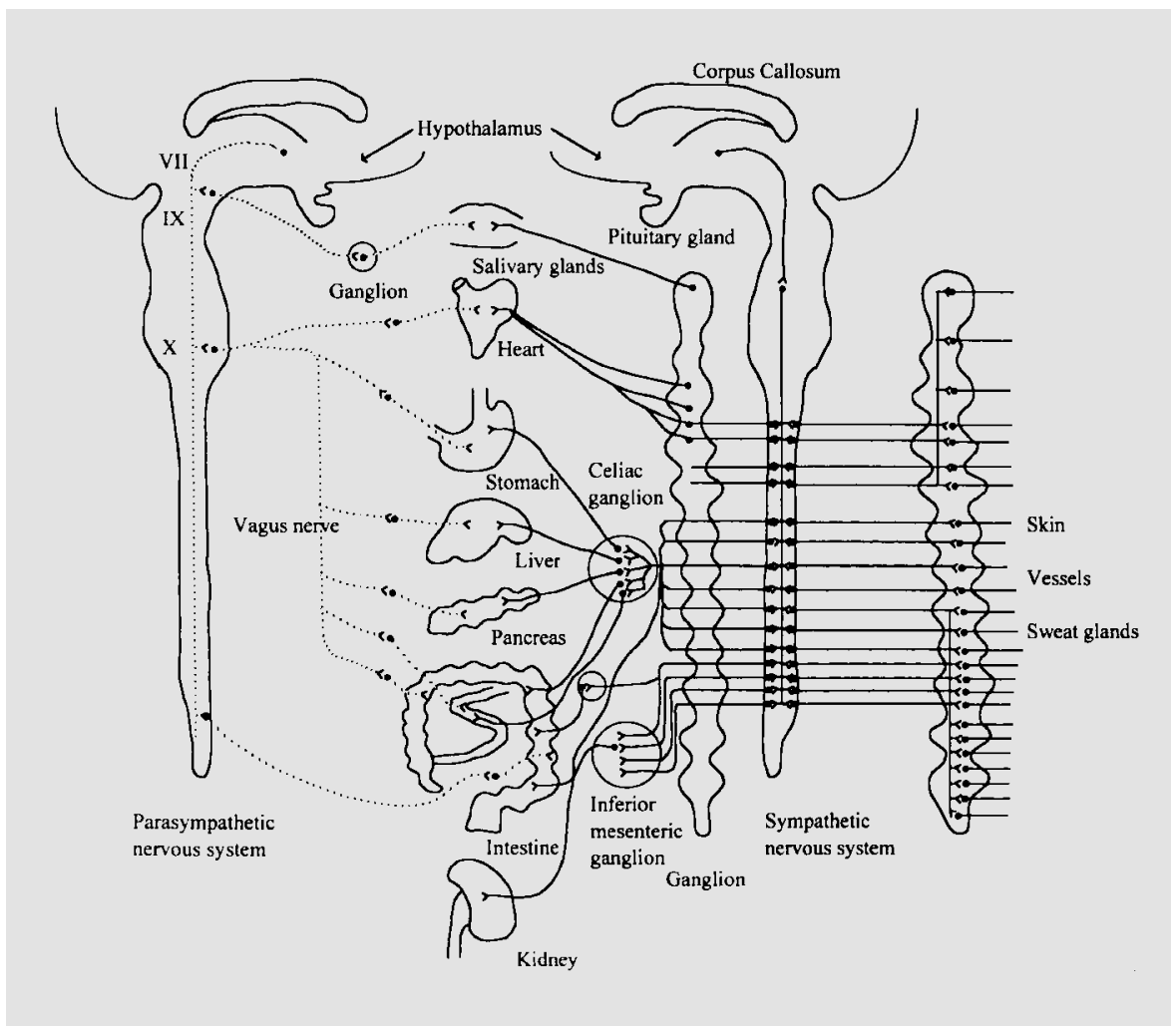
nomic nervous system.

\section{Innervation of the Autonomic Nervous System in the Liver and Sprouting in the Neurons of Regenerated Liver}

The hepatic parenchyma has been shown to have parasympathetic and sympathetic innervations [9-11]. However, it is well known that the intrahepatic distribution of nerve fibers is highly species-dependent; also, even within one species, there are notable variations. To reveal the pattern and type of hepatic innervation in different species, Akiyoshi et al. [12] examined the distribution and density of these nerve fibers. These data indicate that there were differences in the patterns of hepatic innervation among rats, golden hamsters, guinea pigs, dogs and humans. The data also show that (1) in rat and hamster livers, hepatic functions may be regulated by both sympathetic and parasympathetic nerves in the portal region; (2) in guinea pig, dog and human livers, they may be regulated by these fibers both in the interlobular region (parasympathetic and sympathetic systems) and in the intraparenchymal region (sympathetic system), and thus, (3) in the guinea pig, dog and human livers, hepatocytes and sinusoidal cells may be innervated by sympathetic nerves. Berthoud et al. [13] investigated the distribution and structure of vagal rat liver innervation. Vagal afferent fibers produced characteristic terminals at three locations in the liver hilus: (1) fine varicose ending preferentially surrounding, but not entering, the numerous peribiliary glands in the larger intra- and extrahepatic bile ducts; (2) large, cup-shaped terminals in almost all paraganglia, and (3) fine varicose endings in the portal vein adventitia. No fibers and terminals were found in the hepatic parenchyma. Varicose vagal efferent fibers were present within the fascicles of the vagal hepatic branch and fine terminallike structures in a small fraction of the paraganglia. No efferents were found to terminate in the hepatic parenchyma or on the few neurons embedded in nerves or paraganglia. With regard to the sympathetic nerve, in the liver tissues from rats, guinea pigs and humans, Fukuda et al. [14] also reported that sympathetic nerve fibers were localized around blood vessels in the portal area and around the central vein. Normal guinea pig and human liver showed intralobular sympathetic fibers, while rat liver did not [15]. Moreover, it was reported that livers of the guinea pig, rabbit and cat have dense innervation of sympathetic nerves [15]. In these animals, nerve terminals are distributed not only in the Glisson's sheath but also deep into the parenchyma. 
Carobi [16] investigated the possible presence of neural sprouting in the vagal afferent neurons of regenerated rat liver after hepatectomy. This experiment was carried out to see if the increase in hepatic parenchyma could provide an adequate stimulus for the sprouting process. Neural sprouting did not occur in regenerated rat liver after 3 weeks. However, Tadokoro [17] previously demonstrated that the speed of axonal growth was approximately $5 \mathrm{~mm} /$ day around the porta hepatis in a rat surgical hepatic degeneration model. Therefore, it may be possible for the regenerating axons sprouting from proximal axonal stumps to reach the hepatic hilus within 3 days after transplantation. Consistent with this, Takahashi et al. [18] reported that liver allografts became extrinsically reinnervated, with the regenerating axons reaching the hepatic hilus 3 days after transplantation in rats and that the process of extrinsic hepatic reinnervation is considered to almost terminate 4 months. Moreover, Philips et al. [19] also reported that the liver can undergo dramatic afferent reinnervation after subdiaphragmatic vagotomy. Furthermore, it was reported that sympathetic fibers were absent from regenerating nodules in a human cirrhotic liver [14].

\section{Autonomic Nervous System and Hepatic Function}

Selective hepatic vagotomy does not disrupt the putative feedback loop between body weight and feeding [20]. However, it has been reported that the vagotomy reduces food intake, water intake and body weight after hepatic vagotomy only in 100-gram rats, but not 200- and 280gram rats $[21,22]$. When 100-gram animals with either hepatic branch vagotomies or sham operations were housed at three different room temperatures (12-17, 17$22,22-27^{\circ} \mathrm{C}$ ), the reduction in weight of vagotomized animals correlated with the lowest room temperature, and food intake was also decreased in direct relation to the temperature [23]. Therefore, the hepatic vagal mechanism modulates food intake in young animals, and such a mechanism may be modulated by the environmental temperature. Moreover, to further characterize the possible effect of hepatic vagotomy on food intake, Langhans et al. [24] offered rats a novel-tasting diet immediately after hepatic branch vagotomy or sham vagotomy and measured food intake as well as diet selection (novel-tasting vs. familiar diet). They found that hepatic branch vagotomy and sham vagotomy had different aversive effects, which led to transient differences in postoperative food intake when diets with a novel and strong taste were presented during the initial postoperative period. Therefore, information conveyed by the hepatic branch of the vagus can apparently lead to a conditioned feeding response. This is evidence for a more subtle role of the liver in the control of food intake than previously thought.

It is well known that a $90 \%$ majority of the fibers in the vagus nerve is afferent [25]. Vagal afferent nerves were reported to contain glucose-sensitive nerve fibers [26], amino acid-sensitive nerve fibers [27], lipid-sensitive nerve fibers [28] or osmosensitive nerve fibers [29]. With regard to efferent nerve fibers, Niijima [30] recorded efferent discharges from nerve filaments dissected from the hepatic branch of the vagus nerve in the rat. It was concluded that changes in the rate of hepatic glycogenesis, which occur in response to changes in blood glucose concentration, were mediated in part by the vagal efferent innervation of the liver.

Corresponding to the different density of innervation among the species, noradrenaline overflow from perfused liver during hepatic nerve stimulation was much higher in the guinea pig than in the rat [31]. Nevertheless, the nerve stimulation elicited an increase in glucose output from rat liver to approximately the same degree as that from guinea pig liver [31]. These observations suggested that in rat liver most of hepatocytes are not activated by direct contact with the sympathetic nerves, but through an indirect mechanism of signal transfer. One of the possible mechanisms is the involvement of intercellular communication via specific channels of gap junction, which exist between adjacent hepatocytes and permit the passage of small molecules and ions [32]. Therefore, to explore the possible role of gap junctions between liver cells in neural regulation of hepatic glucose metabolism, Iwai et al. [33] examined the effects of hepatic nerve stimulation on metabolic and hemodynamic changes in regenerating rat liver. They found that the impaired effects of sympathetic nerve stimulation on glucose metabolism observed in regenerating liver were derived neither from reduced release of noradrenaline nor from depletion of liver glycogen, but rather from transient reduction of gap junction, which assist signal propagation of the nerve action through intercellular communication in rat liver.

Insulin is regarded to be one of the hepatotrophic factors [34], because it exerts direct effects on organelles, stimulates DNA and protein synthesis [35], and its secretion is known to be regulated through the hepatic vagus branch [36]. However, during starvation, the plasma insulin level was reported to decrease after partial hepatectomy in subdiaphragmatic vagotomized rats and hepatic 
Table 1. Comparisons of the effects of subdiaphragmatic vagotomy and hepatic vagotomy compared with sham vagotomy after partial hepatectomy

\begin{tabular}{lll}
\hline & $\begin{array}{l}\text { Subdiaphragmatic } \\
\text { vagotomy }\end{array}$ & $\begin{array}{l}\text { Hepatic } \\
\text { vagotomy }\end{array}$ \\
\hline Restoration of liver weight & $\downarrow$ & $\rightarrow$ \\
Increase in the DNA of regenerating liver & $\downarrow$ & $\rightarrow$ \\
Increase in the protein content of regenerating liver & $\downarrow$ & $\rightarrow$ \\
Rate of hepatic DNA synthesis & $\downarrow$, delay & $\rightarrow$, delay \\
Plasma insulin level during starvation & $\rightarrow$ & $\rightarrow$ \\
Daily food intake after partial hepatectomy & $\downarrow$ & $\rightarrow$ \\
Body weight increase after partial hepatectomy & $\downarrow$ & $\rightarrow$ \\
\hline
\end{tabular}

$\downarrow=$ Suppress; $\rightarrow=$ no effect. vagotomized rats, as in sham-operated rats [37]. Meanwhile, Trabelsi et al. [38] evaluated the effect of a selective hepatic vagotomy on the insulin response in rats fasted for $24 \mathrm{~h}$ when blood glucose levels were or were not maintained by a constant glucose infusion. It was concluded that the decline in plasma glucose concentration during fasting did not totally explain the insulinopenic response to fasting and that the liver, though the mediation of the hepatic vagus nerve, did not seem to contribute to insulinopenia in 24-hour fasted rats. Moreover, it is well known that, in response to insulin, one of hormones, hepatic insulin-sensitizing substance (HISS) is released from the liver to stimulate glucose uptake in skeletal muscle but not liver or gut. Consistent with this, Lautt et al. [39] recently reported that the interruption of the hepatic parasympathetic nerves by surgical denervation or atropine blocked the release of HISS.

With regard to hepatic enzymes, the hepatic and renal scores, total protein, glutamic pyruvic transaminase (GPT), lactate dehydrogenase, total bilirubin, total cholesterol, blood urea nitrogen and creatinine indicated no significant differences between the hepatic vagotomized and sham vagotomized rats. Moreover, because hepatic branch vagotomy had no effect on the concentration of albumin, one of the proteins specially produced in the liver, the vagus nerve must have no role in protein production in the remaining liver. Meanwhile, it was reported that the value of alkaline phosphatase (ALP) in hepatic vagotomized rats was significantly lower than in controls [40]. Reilly et al. [41] observed histologically that cholinergic nerves innervate bile ducts running along the hepatic vasculature. Moreover, electrical stimulation of vagus nerve induced an increase in intracholedochal pressure, suggesting a direct relationship between the vagus nerve and bile secretion [42]. In general, the serum level of ALP in liver diseases is elevated by bile stasis. Therefore, the elevated activity of ALP could be interpreted as resulting from the bile stasis caused by increased intracholedochal pressure due to vagal stimulation. However, more detailed studies on the exact relationship between the vagus nerve and bile secretion are needed.

\section{Autonomic Nervous System and Liver Regeneration}

\section{Vagus Nerve}

In animal models, the weight of the regenerating liver is restored to approximately $80 \%$ of the preoperative weight $72 \mathrm{~h}$ after partial hepatectomy [43]. Restoration of the liver weight is reported to be significantly impaired in subdiaphragmatic vagotomized rats (table 1) [37]. This impairment is associated with a reduction in food intake, because there was no difference in the liver weights between the control rats and the pair-fed subdiaphragmatic vagotomized rats [44]. Subdiaphragmatic vagotomy also causes considerably more loss of body weight than sham vagotomy. The increase in DNA and protein content of the regenerating liver is also suppressed by subdiaphragmatic vagotomy. Moreover, subdiaphragmatic vagotomy delays and suppresses the rate of hepatic DNA synthesis after partial hepatectomy. Consistent with this, the increase in activity of hepatic aspartate transcarbamoylase and thymidine kinase, the key enzymes in synthesis of pyrimidine nucleotides via the de novo and salvage pathways, respectively, during liver regeneration, is significantly suppressed and retarded in subdiaphragmatic vagotomized rats. The onset of DNA synthesis triggered by partial hepatectomy is also delayed by the subdiaphragmatic vagotomy.

Hepatic vagotomy delays but does not suppress the increase in the rate of hepatic DNA synthesis and the 
activity of thymidine kinase after partial hepatectomy (table 1) [45]. The time-course of liver DNA content restoration after partial hepatectomy is not affected by hepatic vagotomy. Hepatic vagotomy does not affect daily food intake or body weight increase after partial hepatectomy. The difference between subdiaphragmatic vagotomy and hepatic vagotomy depends on the fact that the vagus nerve innervates not only the liver but also many organs [46], and subdiaphragmatic vagotomy, not hepatic vagotomy, reduces food intake and massively delays gastric emptying [44, 47].

Pietroletti et al. [48] examined the relationship of the autonomic nervous system and dual blood supply for an optimal regenerative response in liver remnants after liver resection. They found that the autonomic nerve supply of the portal triad plays at least an important permissive role in liver regeneration. Therefore, there may be the possibilities that the hepatic vagus nerve affects hepatocytes through free radical injury that might be induced by ischemia from vagally induced changes in vascular tone. However, in rats subjected to portacaval transposition in order to increase the liver blood flow, tritiated thymidine was incorporated into liver DNA much as it was in the control rats [49]. Moreover, in examination of acute changes, sectioning of the hepatic branch of splanchnic or vagus nerve did not change the blood flow in the hepatic artery or the portal vein [40]. Alteration in blood supply to the liver associated with nerve sectioning play an insignificant role in liver regeneration. Therefore, the vagus nerve may stimulate liver regeneration directly and specially, although elucidation of its mechanism awaits further studies.

\section{Sympathetic Nerve}

Hepatic branch sympathectomy proved to have no influence on liver regeneration [40]. Moreover, Kato and Shimazu [37] reported that bilateral major splanchnicectomy at the subdiaphragmatic level produced no effect on liver regeneration. However, it has been suggested that adrenergic agents regulate liver regeneration [50]. Cruise et al. [51] also reported that noradrenaline stimulated DNA synthesis by cultured rat hepatocytes through $\alpha_{1-}$ adrenergic receptors. Moreover, Iwai and Shimazu [52] used slightly injured, galactosamine-treated, isolated rat liver and found that the activation of hepatic sympathetic nerves and circulating catecholamines exaggerates acute liver damage through an action on liver cells, which depends on the influx of extracellular $\mathrm{Ca}^{2+}$. In 1960, Calvert and Brody [53] proposed that carbon tetrachloride $\left(\mathrm{CCl}_{4}\right)$ exerted its necrotic effect not by acting on the liver parenchyma directly, but by causing a persistent sympathetic discharge resulting in diminished hepatic blood flow and cellular hypoxia. However, recently, $\mathrm{CCl}_{4}$ is generally proposed to induce cellular injury through its metabolites that are generated by a cytochrome P-450dependent step [54]. Hsu [55] examined effects of the sympathetic nervous system on liver injury induced experimentally by $\mathrm{CCl}_{4}$ in spontaneously hypertensive rats (SHR) and normotensive Wistar-Kyoto rats (WKY). It was found that the SHR had an elevated catecholamine content in the adrenal gland without any treatment, and fluorescence histochemistry also revealed dense adrenergic innervations in the liver. Moreover, the SHR showed greater sensitivity to $\mathrm{CCl}_{4}$ stimulation in the sympathetic nervous system than the WKY, resulting in decreased hepatic blood flow in the acute stage and depleted catecholamine in the adrenal gland and lowered blood pressure in the chronic stage. Plasma GPT activity was increased in both strains but more significantly in the SHR than in the WKY. Histological examination of the liver in the SHR showed established cirrhosis, whereas only bridging fibrosis was seen in the WKY. Therefore, the pathogenesis of the liver damage increased by $\mathrm{CCl}_{4}$ in the SHR is attributable to the enhanced response of the sympathetic nervous system that releases massive amounts of catecholamine which then leads to vasoconstriction and metabolic changes that promote liver damage.

With regards to liver regeneration after partial hepatectomy, to determine if the sympathetic nerve has a role in liver regeneration after partial hepatectomy, Iwai et al. [56] measured the alterations of tissue noradrenaline content and its turnover in rats. Fractional turnover rate of noradrenaline was reduced transiently in regenerating liver 24-48 $\mathrm{h}$ after partial hepatectomy and returned to normal 8-9 days after the operation; therefore, sympathetic nerve activity of the liver was suppressed at the early stage of regeneration. However, Knopp et al. [57] reported that $20 \mathrm{~min}$ after partial hepatectomy, the plasma noradrenaline level increased, but after $1 \mathrm{~h}$, it decreased to the basal level in both partial hepatectomized rats and sham-operated rats and remained unchanged 4 and $24 \mathrm{~h}$ later. Moreover, the plasma levels of adrenaline and corticosterone also rapidly increased $20 \mathrm{~min}$ after partial hepatectomy or sham operation [57]. The first peak of catecholamine and corticosterone levels resulted from an unspecific stressor associated with the surgery. Then at $4 \mathrm{~h}$, the plasma level of adrenaline was higher than in sham-operated rats, but after $24 \mathrm{~h}$, the adrenaline level returned to basal values [57]. The prolonged elevation of adrenaline found after 
partial hepatectomy seems to be specific for liver regeneration.

Ohtake [58] examined the role of the abdominal sympathetic nervous system in regulating portal venous flow in anesthetized rats using an ultrasonic volume flowmeter. Results showed that sympathetic nerve regulated portal venous flow directly. Neither proper hepatic arterial occlusion nor partial hepatecomy affected the response. In the partially hepatectomized animals, stimulation of the hepatic branch did not decrease the splenic flow but decreased the superior mesenteric venous flow and induced a similar response in portal venous flow even when the superior mesenteric venous flow was interrupted. This fact may be related to the possibility that changes in the portal venous flow by the sympathetic nervous system may affect liver regeneration. However, Ohtake et al. [40] also reported that sectioning of sympathetic nerves, which results in increased blood flow in the liver, did not affect liver regeneration.

With regards to immunology, it is well known that thymic atrophy is always induced $24 \mathrm{~h}$ after partial hepatectomy as a stress-associated response. Consistent with this, Minagawa et al. [59] proposed the possibility that sympathetic nerve stimulation is quite important for the induction of the specific pattern of the immune system seen after partial hepatectomy. Sympathetic nerve stimulation after partial hepatectomy results in subsequent activation of natural killer cells, and these natural killer cells might be associated with immunologic surveillance during hepatocyte regeneration.

It is also well known that gentle organ manipulation rapidly causes disturbances in the activation of Kupffer cells [60]. Consistent with this, Schemmer et al. [60] recently reported that both the autonomic nervous system and gut-derived endotoxin were involved in this matter.

\section{Autonomic Nervous System and Liver Apoptosis}

The liver can precisely regulate its growth and mass. Apoptosis is a type of cell death that serves to eliminate excessive or unwanted cells during the remodeling process in liver regeneration, as well as in embryonic development and wound healing [61]. Surgical resection of hepatic lobes or hepatocytes loss caused by viral or chemical injury triggers hepatocyte replication, while enlarged liver mass is corrected by apoptosis. As has been mentioned, noradrenaline markedly enhances the stimulation of hepatic proliferation [51]. However, it was reported that noradrenaline prevented Fas-induced hepatocyte apoptosis via a $\beta_{2}$-adrenergic receptor [62]. These findings led us to speculate that the autonomic nervous system may play a role as a stimulator of cell growth through inhibiting apoptosis in the regenerating liver. Moreover, although it was well known that $\mathrm{CCl}_{4}$ hepatotoxicity was more severe in SHR showing sympathetic hyperactivity than in WKY with normal sympathetic hyperactivity [55], Hamasaki et al. [63] reported that both acute and chronic $\mathrm{CCl}_{4-}$ induced liver damage was enhanced in SHR as compared with WKY. The proliferating cell nuclear antigen (PCNA) labeling index, indicating the number of regenerating hepatocytes, paralleled the severity of liver damage. In contrast, the number of TdT-dUTP nick-end labeling (TUNEL)-positive cells did not correspond to the severity of liver damage. Therefore, Hamasaki et al. [63] suggested that noradrenaline suppressed apoptosis in hepatocytes but did not suppress liver damage.

The hepatic density of $\beta_{2}$-adrenoreceptors of C57BL/6 mice is low but that of recently described transgenic F28 mice is much higher [64]. F28 mice are naturally protected against hamster anti-mouse Fas antigen monoclonal antibody, Jo2; F28 mice were resistant to Jo2-induced liver apoptosis and death. The $\beta$-adreneroreceptor antagonist propranolol sensitized the F28 livers to Jo2. In normal mice, clenbuterol, a $\beta_{2}$-adreneroreceptor-specific agonist, considerably reduced Jo2-induced liver apoptosis and death; salbutamol, another $\beta_{2}$-adreneroreceptor-selective agonist, also reduced Jo2-induced apoptosis and retarded death but with less efficacy than clenbuterol; and propranolol blocked the protective effect of clenbuterol. These indicate that the expression level of functional $\beta_{2^{-}}$ adreneroreceptors modulates Fas-regulated liver apoptosis and that this apoptosis can be inhibited in vivo by giving $\beta_{2}$-adrenergic receptor agonists [62].

\section{Central Nervous System and Liver Regeneration and A poptosis}

The hypothalamus has a vital role in the integration of neurohumoral information [65], and it possesses autonomic centers connected to viscera via the autonomic nervous system. The lateral hypothalamus (LH) and the ventromedial hypothalamus (VMH) nucleus have been studied for their role in the integration of neurohumoral information [66]. Both are considered to act reciprocally in several regulatory functions: feeding behavior, autonomic regulation and metabolic regulation [65]. The $\mathrm{LH}$ is part of the parasympathetic system, and the $\mathrm{VMH}$ 
belongs to the sympathetic system [67, 68] (fig. 2). Lesions in the LH reportedly induced an increase in sympathetic nerve activity, while those in the VMH produced facilitation of vagus nerve activity [23, 69]. As described above, both the sympathetic and parasympathetic nervous system are important modulators of liver regulators [45, 50, 70]. Iwai and Shimazu [71] studied the effects of hypothalamic stimulation on experimental liver injury induced by $\mathrm{CCl}_{4}$ or dimethylnitrosamine in rats by measuring plasma GPT activity as an index of acute liver injury. Electrical stimulation of the $\mathrm{VMH}$ in $\mathrm{CCl}_{4}$-treated rats caused a marked increase in plasma GPT activity, accompanied by a significant decrease in GPT activity in the liver, although $\mathrm{CCl}_{4}$ treatment alone had no significant effect on plasma GPT activity. A similar effect of VMH stimulation on plasma GPT activity was observed in rats treated with dimethylnitrosamine, another hepatotoxic chemical. No such exaggerated effect of VMH stimulation on plasma GPT activity was observed after stimulation of the LH. Surgical sympathetic denervation of the liver greatly suppressed the increase in plasma GPT activity after $\mathrm{CCl}_{4}$ injection and $\mathrm{VMH}$ stimulation. Measurement of regional blood flow indicated that VMH stimulation did not produce a significant change in blood flow to the liver. Therefore, VMH was involved in the progress of chemically-induced liver injury through activation of the sympathetic hepatic nerves, possibly by affecting liver metabolism rather than blood flow to the liver. Moreover, Kiba et al. [72-74] previously reported that vagal activity produced by VMH lesions produced an increase in cell proliferation of the liver. It was also reported that VMH lesions facilitated hepatic regeneration after partial hepatectomy [75]. Although, as mentioned above, previous studies also indicated a reciprocity in the regeneration of autonomic function between the VMH and LH [69], Kiba et al. [76] reported that $\mathrm{LH}$ lesions facilitated hepatic regeneration. The LH has been shown to project directly and strongly to the dorsal motor nucleus of the vagus nerve in the medulla oblongata [65] (fig. 2), and is part of the parasympathetic nervous system [67]. Iwai and Shimazu [56] measured the changes in hepatic noradrenergic content and its turnover after partial hepatectomy to determine whether the sympathetic nerve has a role in liver regeneration; they found that sympathetic nerve activity of the liver was suppressed early (24-48 h) after partial hepatectomy. In contrast, an increase in sympathetic firing rate occurred $24 \mathrm{~h}$ after LH lesioning [77]. Therefore, it was suggested that LH lesions may prevent the suppression of sympathetic nerve activity soon after partial hepatectomy, thereby facilitating hepatic DNA synthesis.

Liver Regeneration, Apoptosis and

Autonomic Nervous System

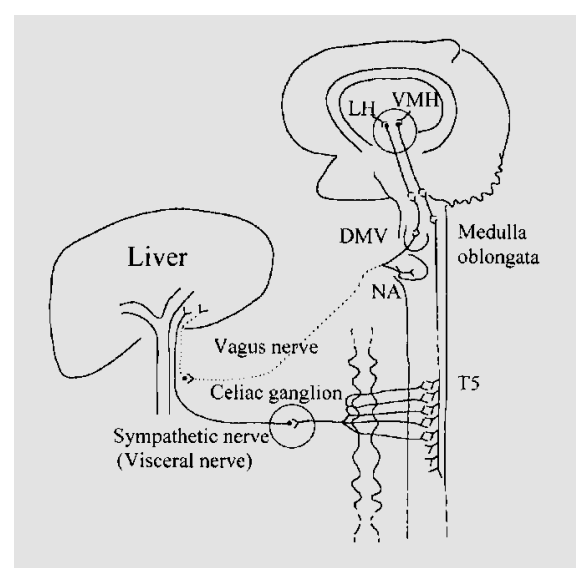

Fig. 2. Relationships between hepatic autonomic nervous system and central nervous system. DMV = nucleus dorsalis nervi vagi; $\mathrm{LH}=$ lateral hypothalamus; $\mathrm{NA}=$ nucleus ambiguus; $\mathrm{VMH}=$ ventromedial hypothalamus.

Central neuropeptides play important roles in many instances of physiological and pathophysiological regulation mediated through the autonomic nervous system. In regard to the hepatobiliary system, several neuropeptides act in the brain to regulate bile secretion, hepatic blood flow, and hepatic proliferation. Stressors and sympathetic nerve activation are reported to exacerbate experimental liver injury $[55,78,79]$. Some stressors are known to stimulate corticotropin-releasing factor (CRF) synthesis in the central nervous system and induce activation of sympathetic nerves in animal models [80-82]. Yokohama et al. [83] examined the effect of intracisternal CRF on $\mathrm{CCl}_{4}$ induced acute liver injury in rats. Intracisternal injection of CRF dose-dependently enhanced elevation of the serum GPT level induced by $\mathrm{CCl}_{4}$. Elevation of serum GPT, ALP and total bilirubin levels by $\mathrm{CCl}_{4}$ were also enhanced by intracisternal $\mathrm{CRF}$ injection. It was found that CRF acted in the brain to exacerbate acute liver injury through the sympathetic-noradrenergic pathways. Moreover, Yoneda et al. [84] studied the effect of thyrotropin-releasing hormone (TRH) on hepatic DNA synthesis in rats. TRH is distributed throughout the central nervous system and acts as a neurotransmitter to regulate gastric functions through the vagus nerve. It was found that TRH acted in the central nervous system to stimulate hepatic DNA synthesis through vagal and cholinergic mechanisms and that TRH might be the chemical messenger involved in brain regulation of hepatic proliferation.

Digestion 2002;66:79-88 


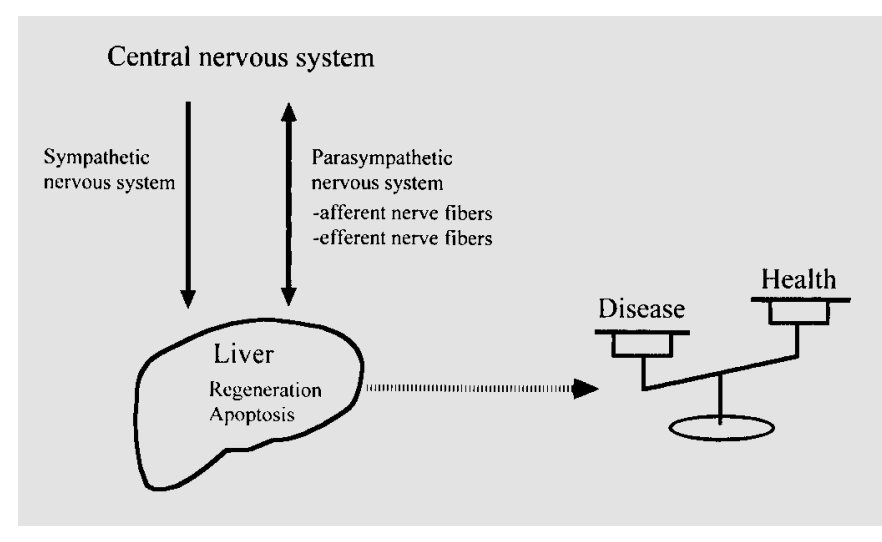

Fig. 3. Schema of hepatic autonomic nervous system and liver disease.

$\gamma$-Aminobutyric acid (GABA) is a potent inhibitory neurotransmitter with growth-regulatory properties. In fulminant hepatic failure, a condition in which hepatic regeneration may be impaired, systemic serum GABA concentrations are markedly elevated. Minuk et al. [85] determined whether increased amounts of circulating GABA interfere with hepatic regenerative activity. Their results indicated that elevated serum GABA concentrations interfered with the hypertrophic component of hepatic regeneration following partial hepatectomy in rats. Recently, Biju et al. [86] reported that GABAergic activity served to maintain hepatocytes in a quiescent state. Moreover, Biju et al. [86] investigated GABAergic alterations in the hypothalamus and plasma noradrenaline levels during compensatory hyperplasia after partial hepatectomy, lead nitrate-induced direct hyperplasia, and N-nitro- sodiethylamine-induced neoplasia in liver. The results indicated that liver cell proliferation influences hypothalamic GABAergic neurotransmission, and these changes regulate hepatic proliferation through sympathetic stimulation.

With regards to liver apoptosis, Kiba et al. [87] recently examined whether the Fas (APO-1/CD95)/Fas ligand system mediates apoptosis in rats with $\mathrm{VMH}$ lesions. The results indicated that vagal firing after VMH lesioning may stimulate Fas/Fas ligand system-mediated apoptosis through the cholinergic system in the rat liver. However, Fujimoto et al. [88] showed that intestinal mucosal apoptosis decreased in the VMH-lesioned rat. Therefore, further investigations may be required to determine the mechanisms by which VMH lesions modulate apoptosis.

\section{Conclusions}

Current literature indicates that the autonomic nervous system regulates liver regeneration and apoptosis. Moreover, the central nervous system modulates them through the autonomic nervous system. Recently, there has been increasing interest in the relationship between liver disease and autonomic nervous system (fig. 3). In regard to this matter, future studies should outline a more complete mechanism.

\section{Acknowledgement}

This work was supported in part by Grant 09670562 from the Ministry of Education of Japan.

\section{References}

1 Berthoud HR, Neuhuber WL: Functional and chemical anatomy of the afferent vagal system. Auton Neurosci 2000;85:1-17.

2 Fausto N: Liver regeneration. J Hepatol 2000; 32(suppl 1):19-31.

3 Jungermann K, Stumpel F: Role of hepatic, intrahepatic and hepatoenteral nerves in the regulation of carbohydrate metabolism and hemodynamics of the liver and intestine. Hepatogastroenterology 1999;46(suppl 2):1414-1417.

4 Rangari M, Sinha S, Kapoor D, Mohan JC, Sarin SK: Prevalence of autonomic dysfunction in cirrhotic and noncirrhotic portal hypertension. Am J Gastroenterol 2002;97:707713.
5 Hendrickse MT, Thuluvath PJ, Triger DR: Natural history of autonomic neuropathy in chronic liver disease. Lancet 1992;339:14621464.

6 Dillon JF, Plevris JN, Nolan J, Ewing DJ, Neilson JM, Bouchier IA, Hayes PC: Autonomic function in cirrhosis assessed by cardiovascular reflex tests and 24-hour heat rate variability. Am J Gastroenterol 1994;89:1544-1547.

7 Miyajima H, Nomura M, Muguruma N, Okahisa $T$, Shibata $H$, Okamura $S$, Honda $H$, Shimizu I, Harada M, Saito K, Nakaya Y, Ito S: Relationship among gastric motility, autonomic activity, and portal hemodynamics in patients with liver cirrhosis. J Gastroenterol Hepatol 2001;16:647-659.
8 Pozzi M, Grassi G, Redaelli E, Dell'oro R, Ratti L, Redaelli A, Foglia G, Di Lelio A, Mancia G: Patterns of regional sympathetic nerve traffic in preascitic and ascitic cirrhosis. Hepatology 2001;34:1113-1118.

9 Nobin A, Baumgarten HG, Falck B, Ingemansson S, Moghimzadeh E, Rosengren E, Fausto $\mathrm{N}$ : Organization of the sympathetic innervation in liver tissue from monkey and man. Cell Tissue Res 1978;195:371-380.

10 Carobi C, Magni F: The afferent innervation of the liver: A horseradish peroxidase study in the rat. Neurosci Lett 1981;23:269-274.

11 Rogers RC, Hermann GE: Central Connections of the hepatic branch of the vagus nerve: A horseradish peroxidase histochemical study. J Auton Nerv Syst 1983;7:165-174. 
12 Akiyoshi H, Gonda T, Terada T: A comparative histochemical and immunohistochemical study of aminergic, cholinergic and peptidergic innervation in rat, hamster, guinea pig, dog and human livers. Liver 1998;18:352-359.

13 Berthoud HR, Kressel M, Neuhuber WL: An anterograde tracing study of the vagal innervation of rat liver, portal vein and biliary system. Anat Embryol (Berl) 1992;186:431-442.

14 Fukuda Y, Imoto M, Koyama Y, Miyazawa Y, Hayakawa T: Demonstration of noradrenalineimmunoreactive nerve fibres in the liver. J Int Med Res 1996;24:466-472.

15 Metz W, Forssmann WG: Communications of Liver Cells. Lancaster, MTP Press, 1980, pp 121-127.

16 Carobi C: Vagal afferent innervation in regenerated rat liver. Experientia 1990;46:50-53

17 Tadokoro F: Immunohistochemical study on the regeneration of nerve fibers of rat liver after surgical severance (in Japanese). Acta Hepatol Jap 1994;35:29-38.

18 Takahashi T, Kakita A, Sakamoto I, Takahash Y, Hayashi K, Tadokoro F, Yamashita S: Immunohistochemical and electron microscopic study of extrinsic hepatic reinnervation following orthotopic liver transplantation in rats. Liver 2001;21:300-308.

19 Phillips RJ, Baronowsky EA, Powley TL: Regenerating vagal afferents reinnervate gastrointestinal tract smooth muscle of the rat. J Comp Neuro1 2000;421:325-346.

20 Johnston DG, Johnson GA, Alberti KG, Millward-Sadler GH, Mitchell J, Wright R: Hepatic regeneration and metabolism after partial hepatectomy in normal rats: Effects of insulin therapy. Eur J Clin Invest 1986;16:384-390.

21 Sakaguchi T, Yamazaki M, Tamaki M, Niijima A: Changes in food intake after hepatic vagotomy at a stage of development in rats. Neurosci Lett 1985;61:317-320.

22 Sakaguchi T, Yamazaki M: Changes in water intake following hepatic vagotomy in young rats. J Auton Nerv Syst 1986;17:243-246.

23 Sakaguchi T, Tamaki M: The body weight loss of young rats caused by hepatic branch vagotomy is influenced by environmental temperature. Physiol Behav 1988;43:673-675.

24 Langhans W, Kunz U, Scharrer E: Hepatic vagotomy increases consumption of a noveltasting diet presented immediately after surgery. Physiol Behav 1989;46:671-678.

25 Sawchenko PE, Friedman MI: Sensory functions of the liver. Am J Physiol 1979;236:R5R20.

26 Sakaguchi T, Iwanaga M: Effects of $D$-glucose anomers on afferent discharge in the hepatic vagus nerve. Experientia 1982;38:475-476.

27 Torii K, Niijima A: Effects of lysine on afferent activity of the hepatic branch of the vagus nerve in normal and $L$-lysine-deficient rats. Physiol Behav 2001;72:685-690.

28 Randich A, Spraggins DS, Cox JE, Meller ST, Kelm GR: Jejunal or portal vein infusions of lipids increase hepatic vagal afferent activity. Neuroreport 2001;12:3101-3105.
29 Niijima A: Afferent discharges from osmoreceptors in the liver of the guinea pig. Nutrition 1996;12:392-393.

30 Niijima A: Blood glucose levels modulate efferent activity in the vagal supply to the rat liver. $\mathbf{J}$ Physiol 1985;364:105-112.

31 Jungermann K, Gardemann A, Beuers U, Balle C, Sannemann J, Beckh K, Hartmann H: Regulation of liver metabolism by the hepatic nerves. Adv Enzyme Regul 1987;26:63-88.

32 Loewenstein WR: Junctional intercellular communication and the control of growth. Biochim Biophys Acta 1979;560:1-65.

33 Iwai M, Miyashita T, Shimazu T: Inhibition of glucose production during hepatic nerve stimulation in regenerating rat liver perfused in situ. Possible involvement of gap junctions in the action of sympathetic nerves. Eur $\mathrm{J}$ Biochem 1991;200:69-74.

34 Leffert HL, Koch KS, Moran T, Rubalcava B: Hormonal control of rat liver regeneration. Gastroenterology 1979;76:1470-1482.

35 Goldfine ID: Does insulin need a second messenger? Diabetes 1977;26:148-155.

36 Yamazaki M, Sakaguchi T: Pancreatic vagal functional distribution in the secretion of insulin evoked by portal infusion of $D$-glucose. Brain Res 1989;484:357-360.

37 Kato H, Shimazu T: Effect of autonomic denervation on DNA synthesis during liver regeneration after partial hepatectomy. Eur J Biochem 1983; 134:473-478.

38 Trabelsi F, Helie R, Lavoie JM: Effect of hepatic vagotomy on plasma insulin levels during fasting in the rats. Physiol Behav 1995;58: 1111-1115.

39 Lautt WW, Macedo MP, Sadri P, Takayama S, Duarte Ramos F, Legare DJ: Hepatic parasympathetic (HISS) control of insulin sensitivity determined by feeding and fasting. Am J Physiol 2001;281:G29-G36.

40 Ohtake M, Sakaguchi T, Yoshida K, Muto T: Hepatic branch vagotomy can suppress liver regeneration in partially hepatectomized rats. HPB Surg 1993;6:277-286.

41 Reilly FD, McCuskey PA, McCuskey RS, Kato $\mathrm{H}$, Shimazu T: Intrahepatic distribution of nerves in the rat. Anat Rec 1978;191:55-67.

42 Madrid JA, Salido GM, Martinez de Victoria E: Effect of the antimuscarinic agent pirenzepine on the in vivo biliary secretion of dogs in response to various stimuli. Physiol Bohemoslov 1988;37:67-77.

43 Kountouras J, Boura P, Lygidakis NJ: Liver regeneration after partial hepatectomy. Hepatogastroenterology 2001;48:556-562.

44 Gortz L, Bjorkman AC, Andersson H, Kral JG: Truncal vagotomy reduces food and liquid intake in man. Physiol Behav 1990;48:779-781.

45 Tanaka K, Ohkawa S, Nishino T, Niijima A, Inoue $\mathrm{S}$ : Role of the hepatic branch of the vagus nerve in liver regeneration in rats. Am J Physiol 1987;253:G439-G444.

46 Kraly FS, Jerome C, Smith GP: Specific postoperative syndromes after total and selective vagotomies in the rat. Appetite 1986;7:1-17.
47 Houghton AD, Liepins P, Clarke SM, Mason RC: Effect of gastric resection, Roux-en-Y diversion and vagotomy on gastric emptying in the rat. Br J Surg 1994;81:75-80.

48 Pietroletti R, Chamuleau RA, Speranza V, Lygidakis NJ: Immunocytochemical study of the hepatic innervation in the rat after partial hepatectomy. Histochem J 1987;19:327-332.

49 Blumgart LH: Liver atrophy, hypertrophy and regenerative hyperplasia in the rat: The relevance of blood flow; in Porter R, Whelan $\mathrm{J}$ (eds): Ciba Found Symp 55 (NS): Hepatotrophic Factors. Amsterdam, Elsevier Excerpta Medica, 1978, pp 181-215.

50 Morley CG, Royse VL: Adrenergic agents as possible regulators of liver regeneration. Int $\mathrm{J}$ Biochem 1981;13:969-973.

51 Cruise JL, Knechtle SJ, Bollinger RR, Kuhn C, Michalopoulos G: Alpha-1-adrenergic effects and liver regeneration. Hepatology 1987;7: 1189-1194.

52 Iwai M, Shimazu T: Exaggeration of acute liver damage by hepatic sympathetic nerves and circulating catecholamines in perfused liver of rats treated with $D$-galactosamine. Hepatology 1996;23:524-529.

53 Calvert DN, Brody TM: Role of the sympathetic nervous system in $\mathrm{CC} 14$ hepatotoxicity. Am J Physiol 1960;198:669-676.

54 Berger ML, Bhatt H, Combes B, Estabrook RW: CC14-induced toxicity in isolated hepatocytes: The importance of direct solvent injury. Hepatology 1986;6:36-45.

55 Hsu CT: The role of the sympathetic nervous system in promoting liver cirrhosis induced by carbon tetrachloride, using the essential hypertensive animal (SHR). J Auton Nerv Syst 1992; 37:163-173.

56 Iwai M, Shimazu T: Alteration in sympathetic nerve activity during liver regeneration in rats after partial hepatectomy. J Auton Nerv Syst 1992;41:209-214.

57 Knopp J, Jezova D, Rusnak M, Jaroscakova I, Farkas R, Kvetnansky R: Changes in plasma catecholamine and corticosterone levels and gene expression of key enzymes of catecholamine biosynthesis in partially hepatectomized rats. Endocr Regul 1999;33:145-153.

58 Ohtake M: The role of the abdominal sympathetic nervous system in regulating portal venous flow and its functional distribution. Surg Today 1992;22:128-136.

59 Minagawa M, Oya H, Yamamoto S, Shimizu T, Bannai M, Kawamura H, Hatakeyama K, Abo T: Intensive expansion of natural killer T cells in the early phase of hepatocyte regeneration after partial hepatectomy in mice and its association with sympathetic nerve activation. Hepatology 2000;31:907-915.

60 Schemmer P, Enomoto N, Bradford BU, Bunzendahl H, Raleigh JA, Thurman RG: Autonomic nervous system and gut-derived endotoxin: Involvement in activation of Kupffer cells after in situ organ manipulation. World $\mathrm{J}$ Surg 2001;25:399-406.

61 Lockshin RA, Zakeri ZF: Programmed cell death: New thoughts and relevance to aging. J Gerontol 1990;45:B135-B140. 
62 Andre C, Couton D, Gaston J, Erraji L, Renia L, Varlet P, Briand P, Guillet JG: Beta-2adrenergic receptor-selective agonist clenbuterol prevents Fas-induced liver apoptosis and death in mice. Am J Physiol 1999;276:G647G654.

63 Hamasaki K, Nakashima M, Naito S, Akiyama Y, Ohtsuru A, Hamanaka Y, Hsu CT, Ito M, Sekine I: The sympathetic nervous system promotes carbon tetrachloride-induced liver cirrhosis in rats by suppressing apoptosis and enhancing the growth kinetics of regenerating hepatocytes. J Gastroenterol 2001;36:111120.

64 Andre C, Erraji L, Gaston J, Grimber G, Briand P, Guillet JG: Transgenic mice carrying the human $\beta_{2}$-adrenergic receptor gene with its own promoter overexpress $\beta_{2}$-adrenergic receptors in liver. Eur J Biochem 1996;241:417424.

65 Oomura Y, Yoshimatsu H: Neural network of glucose monitoring system. J Auton Nerv Syst 1984;10:359-372.

66 Okamoto K, Yamori Y, Oshima A, Tanaka T: Development of substrains in spontaneously hypertensive rats: Genealogy, isozymes and effect of hypercholesterolemic diet. Jpn Circ J 1972;36; 461-470.

67 Ban T: Fiber connections in the hypothalamus and some autonomic functions. Pharmacol Biochem Behav 1975;3(suppl 1):3-13.

68 Niijima A: Nervous regulation of metabolism. Prog Neurobiol 1989;33:135-147.

69 Yoshimatsu H, Niijima A, Oomura Y, Katafuchi T: Lateral and ventromedial hypothalamic influences on hepatic autonomic nerve activity in the rat. Brain Res Bull 1988;21:239-244.

70 Lamar C Jr, Holloway LS Jr: The effect of vagotomy on hepatic regeneration in rats. Acta Hepatogastroenterol (Stuttg) 1977;24:7-10
71 Iwai M, Shimazu T: Effects of ventromedial and lateral hypothalamic stimulation on chemically-induced liver injury in rats. Life Sci 1988;42:1833-1840.

72 Kiba T, Tanaka K, Inoue S, Endo O, Takamura Y: Comparison of DNA contents of visceral organs in rats with ventromedial hypothalamic lesions and a high fat diet. Neurosci Lett 1991; 126:127-130.

73 Kiba T, Tanaka K, Endo O, Inoue S: Role of vagus nerve in increased DNA synthesis after hypothalamic ventromedial lesions in rat liver. Am J Physiol 1992;262:G483-G487.

74 Kiba T, Tanaka K, Numata K, Saito S, Sekihara $\mathrm{H}$ : Hepatocyte proliferation in rats after ventromedial hypothalamic lesions: Immunoreactivity patterns of proliferating cell nuclear antigen. J Gastroenterol 1998;33:523-528.

75 Kiba T, Tanaka K, Numata K, Hoshino M, Inoue $\mathrm{S}$ : Facilitation of liver regeneration after partial hepatectomy by ventromedial hypothalamic lesions in rats. Pflügers Arch 1994;428: 26-29.

76 Kiba T, Tanaka K, Inoue S: Lateral hypothalamic lesions facilitate hepatic regeneration after partial hepatectomy in rats. Pflügers Arch 1995;430:666-671.

77 Sakaguchi T, Takahashi M, Bray GA: Lateral hypothalamus and sympathetic firing rate. Am J Physiol 1988;255:R507-R512.

78 Fukudo S, Suzuki J, Tanaka Y, Iwahashi S, Nomura T: Impact of stress on alcoholic liver injury: A histopathological study. J Psychosom Res 1989;33:515-521.

79 Hsu CT, Schichijo K, Ito M, Sekine I: The effect of chemical sympathectomy on acute liver injury induced by carbon tetrachloride in spontaneously hypertensive rats. J Auton Nerv Syst 1993;43:91-96.

80 Kalin NH, Takahashi LK, Chen FL: Restraint stress increases corticotropin-releasing hormone mRNA content in the amygdala and paraventricular nucleus. Brain Res 1994;656: 182-186.
81 Monnikes H, Raybould HE, Schmidt B, Tache Y: CRF in the paraventricular nucleus of the hypothalamus stimulates colonic motor activity in fasted rats. Peptides 1993;14:743-747.

82 Monnikes H, Schmidt BG, Tebbe J, Bauer C, Tache Y: Microinfusion of corticotropin-releasing factor into the locus coeruleus/subcoeruleus nuclei stimulates colonic motor function in rats. Brain Res 1994;644:101-108.

83 Yokohama S, Yoneda M, Nakamura K, Makino I: Effect of central corticotropin-releasing factor on carbon tetrachloride-induced acute liver injury in rats. Am J Physiol 1999;276: G622-G628.

84 Yoneda M, Tamori K, Sato Y, Yokohama S, Nakamura K, Kono T, Makino I: Central thyrotropin-releasing hormone stimulates hepatic DNA synthesis in rats. Hepatology 1997;26: 1203-1208.

85 Minuk GY, Gauthier T: The effect of $\gamma$-aminobutyric acid on hepatic regenerative activity following partial hepatectomy in rats. Gastroenterology 1993;104:217-221.

86 Biju MP, Pyroja S, Rajeshkumar NV, Paulose CS: Hypothalamic GABA receptor functional regulation and liver cell proliferation. Mol Cell Biochem 2001;216:65-70.

87 Kiba T, Saito S, Numata K, Kon Y, Mizutani $\mathrm{T}$, Sekihara H: Expression of apoptosis on rat liver by hepatic vagus hyperactivity after ventromedial hypothalamic lesioning. Am J Physiol 2001;280:G958-G967.

88 Fujimoto K, Iwakiri R, Utsumi H, Kojima M, Ishibashi S, Wu B, Sakata H, Noda T: Effect of the central nervous system on mucosal growth and apoptosis in the small intestine. Digestion 2001;63(suppl 1):108-111. 